\title{
Lesión del manguito rotador: diagnóstico, tratamiento y efecto de la facilitación neuromuscular propioceptiva
}

\author{
Rotator cuff injury: diagnosis, treatment and effect \\ of proprioceptive neuromuscular facilitation
}

\author{
Stephanie Castellanos-Madrigal, ${ }^{*, 1}$ Eliel Magdaleno-Navarro,*,2 \\ Valeria Herrera-Rodríguez, ${ }^{*, 3}$ María Dolores García, ${ }^{*, 4}$ Olivia Torres-Bugarín ${ }^{\ddagger}, 5$
}

\begin{abstract}
RESUMEN. Introducción: El manguito rotador es una estructura compleja, conformada por cuatro músculos y tendones unidos a la articulación del hombro. Aquí se genera la fuerza que produce el movimiento y la estabilidad. Las lesiones son la causa más frecuente de dolor y limitación funcional del hombro y se asocian a factores extrínsecos como traumatismos de alta energía o deportes de contacto, así como factores intrínsecos entre los que se incluye el envejecimiento. Objetivo: Mostrar evidencia de los beneficios del uso de la Facilitación Neuromuscular Propioceptiva (FNM) como tratamiento conservador de elección para las lesiones del manguito rotador. Material y métodos: Se efectuaron búsquedas de artículos en MEDLINE (1995-hasta la actualidad), los términos de búsqueda fueron FNP (Proprioceptive neuromuscular facilitation), manguito rotador (rotator cuff), hombro (shoulder). Se incluyeron los artículos si el título contenía alguno de los descriptores y si eran trabajos originales en los que aplicaron la facilitación neuromuscular propioceptiva. Se excluyeron trabajos de revisión, también aquéllos en los que se aplicó la FNP a lesiones que no fueran de hombro. Resultados: Se localizaron 40 publicaciones que contenían alguno de los descriptores en el título; no obstante, sólo nueve cumplían los criterios de inclusión y exclusión. De estos estudios uno fue de caso clínico, uno con pacientes con capsulitis adhesiva, uno con ruptura del supraespinoso, otro con síndrome de choque subacromial, uno con tendinitis calcificada, otro con lesión crónica, uno de ejercicios rutinarios y otro en deporte de lanzamiento de mano, dos personas sanas y un estudio en personas mayores. En todos los casos se mostró la efectividad del FNP, la cual disminuyó el dolor y mejoró el movimiento y la funcionalidad del hombro, incluso en todas las edades o actividades. Conclusiones: La lesión del manguito rotador es una patología más frecuente en hombro, hoy en día existen múltiples alternativas de tratamientos conservadores, entre las que se incluye FNP, la cual es un método conocido en el ámbito de la fisioterapia; no obstante, pese a sus buenos resultados, no existe suficiente evidencia que sustente su eficiencia en este tipo de lesiones, por lo que
\end{abstract}

${ }^{1}$ http://orcid.org/0000-0002-7541-11179

${ }^{2} \mathrm{http} / / /$ orcid.org/0000-0001-9215-4792

${ }^{3} \mathrm{http}: / /$ orcid.org/0000-0002-5131-462X

${ }^{4}$ http://orcid.org/0000-0002-1861-8250

${ }^{5}$ http://orcid.org/0000-0003-4541-8067

* Escuela de Terapia Física.

‡ Programa Internacional de Medicina.

Universidad Autónoma de Guadalajara.
Recibido: 06 de Febrero de 2020. Aceptado: 27 de Febrero de 2020.

Conflicto de intereses: Los autores de este artículo declaran no tener ningún tipo de conflicto de intereses en relación con la investigación presentada a esta revista, ni recibir beneficio económico alguno por la publicación de este artículo.

Correspondencia:

Dra. en C. Olivia Torres-Bugarín

E-mail: oliviatorres@hotmail.com 
se recomienda promover más su aplicación, investigar y documentar adecuadamente para que un mayor número de personas puedan ser beneficiadas.

Palabras clave: Facilitación neuromuscular propioceptiva, manguito rotador, rehabilitación.

\begin{abstract}
Introduction: The rotator cuff is a complex structure, made up of four muscles and tendons attached to the shoulder joint. Here the force that produces movement and stability is generated. Injuries are the most frequent cause of shoulder pain and functional limitation and are associated with extrinsic factors such as high energy trauma or contact sports, as well as intrinsic factors including aging. Objective: To show evidence of the benefits of using Proprioceptive Neuromuscular Facilitation as a conservative treatment of choice for rotator cuff injuries. Material and method: MEDLINE searches were conducted (1995-present), search terms included facilitación neuromuscular propioceptiva (proprioceptive neuromuscular facilitation therapy), manguito rotador (rotator cuff), hombro (shoulder). The articles were included if the title contained any of the descriptors, and if they were original papers in which they applied proprioceptive neuromuscular facilitation as treatment. Review papers were also excluded, as well as those where Proprioceptive Neuromuscular Facilitation was applied to non-shoulder injuries. Results: 40 publications were found that contained some of the descriptors in the title, however only 9 met the inclusion and exclusion criteria: of these studies one was a clinical case, one with patients with adhesive capsulitis, one with rupture of the supraspinatus, shock syndrome subacromial, one with calcified tendinitis, one with chronic session, one routine exercise and another in hand throwing sport, two healthy people and one study in older people. In all cases, the effectiveness of the PNF was shown, which decreased pain and improved movement and functionality of the shoulder, even at all ages or activities. Conclusions: Rotator cuff injury is a more frequent pathology in the shoulder, today there are multiple alternatives for conservative treatments, including PNF, which is a known method in the field of physiotherapy; however, despite its good results, there is not enough evidence to support its efficiency in this type of injury, so it is recommended to further promote its application and document it properly so that more people can benefit.
\end{abstract}

Keywords: Proprioceptive neuromuscular facilitation, rotator cuff, rehabilitation.

\section{INTRODUCCIÓN}

La facilitación neuromuscular propioceptiva (FNP) es un método para lograr rápida y eficaz mejoría de la flexibilidad; la meta principal de este método es ayudar a los pacientes a alcanzar su nivel de funcionalidad más alto. Se plantea como el mejor enfoque funcional positivo en el camino para estimular a los pacientes y lograr resultados de tratamiento superiores. ${ }^{1}$ Son imprescindibles los movimientos espirales y diagonales que involucran los diferentes planos del espacio. Hoy en día, existen varias formas de realizar los estiramientos y una de ellas es la FNP; ésta es la técnica más eficaz para ganar mayor arco de movilidad y se utiliza para comprobar su efectividad en la ruptura del manguito rotador. Los procedimientos básicos para la FNP proporcionan a los fisioterapeutas las herramientas para ayudar al paciente a conseguir la función motora eficaz. ${ }^{2}$

\section{Anatomía del hombro y del manguito rotador}

El hombro: es la articulación más móvil y menos estable del cuerpo humano. Es una estructura compleja conformada por la parte proximal del húmero, clavícula, escápula y sus uniones con el esternón, la caja torácica y los tejidos blandos. Está constituido por varias articulaciones, entre ellas la esternoclavicular, acromioclavicular, glenohumeral y escapulotorácica, las cuales trabajan juntas a un ritmo sincrónico para permitir el movimiento. ${ }^{3}$ Esta complejidad le confiere la característica de ser una de las articulaciones más móviles del cuerpo; por lo tanto, es sitio de múltiples lesiones y patologías inflamatorias, traumáticas, así como degenerativas. ${ }^{4}$

Manguito rotador: está conformado por un grupo de músculos y tendones que rodea la articulación del hombro y mantiene firme la cabeza del húmero en la cavidad poco profunda del 
hombro. De los cuatro músculos que se insertan en el húmero, el supraespinoso, infraespinoso y redondo menor se insertan juntos en la parte externa, mientras el subescapular se inserta en la parte medial (Figura 1). ${ }^{5}$

\section{Causas de lesión del manguito rotador}

Los desgarros del manguito rotador pueden ocurrir como resultado de una sola lesión y son común en personas que realizan movimientos repetitivos por encima de la cabeza en sus trabajos o deportes; sin embargo, la causa más frecuente de lesión es la mezcla del desgaste normal por edad, debido a que después de los 40 años los tendones se desgarran con mayor facilidad. ${ }^{6}$ Otras causas son algunas enfermedades musculoesqueléticas que debilitan al manguito rotador y predisponen a lesiones. ${ }^{7}$

\section{Diagnóstico de las lesiones de manguito rotador}

El diagnóstico de una lesión de manguito rotador es clínico, se hace mediante la interrogación y exploración física completa, aplicación de pruebas físicas especiales y pruebas auxiliares de laboratorio y gabinete. ${ }^{8}$ En el interrogatorio se pregunta la edad (entre más edad, mayor posibilidad de lesiones de hombro), actividades recreativas con movimientos de lanzamiento, actividades laborales con máquinas que produzcan vibración o que mantenga elevado el brazo por encima del hombro, traumatismos previos en el hombro y la aparición de dolor durante el sueño. ${ }^{9}$ En la exploración física se evaluará la cintura escapular como una estructura global y sus componentes individuales, arcos de movilidad en forma pasiva y activa, también la fuerza muscular, pero poniendo especial énfasis en los movimientos de abducción, flexión, extensión, rotación interna y externa. La combinación de la interrogación y de la exploración física en la evaluación del paciente con lesión del manguito rotador es fundamental para poder establecer el diagnóstico y poder discernir la musculatura afectada de la sana. ${ }^{9}$

\section{Maniobras o pruebas específicas}

Las maniobras o pruebas musculares específicas ayudarán a determinar la estructura en específico que está lesionada, el inconveniente es que son numerosas y pueden producir dolor. Entre las específicas para esta lesión de manguito rotador están:

- Maniobra de Apley superior: valora todo el conjunto del manguito rotador.

- Maniobra de Job: valora al músculo supraespinoso.

- Prueba de flexión de codo posterior: valora al músculo subescapular.

- Prueba de pinzamiento: valora un pinzamiento del tendón del subescapular.

La exploración de los signos especiales de pinzamiento y de lesión de manguito rotador se realizan para orientar al clínico sobre el tipo de lesiones existentes. ${ }^{9}$

\section{Clasificación de las lesiones}

La clasificación de rupturas de la lesión del manguito rotador se puede medir mediante la movilidad y el tamaño: mediante la movilidad la rotura puede ser móvil y reductible o re-

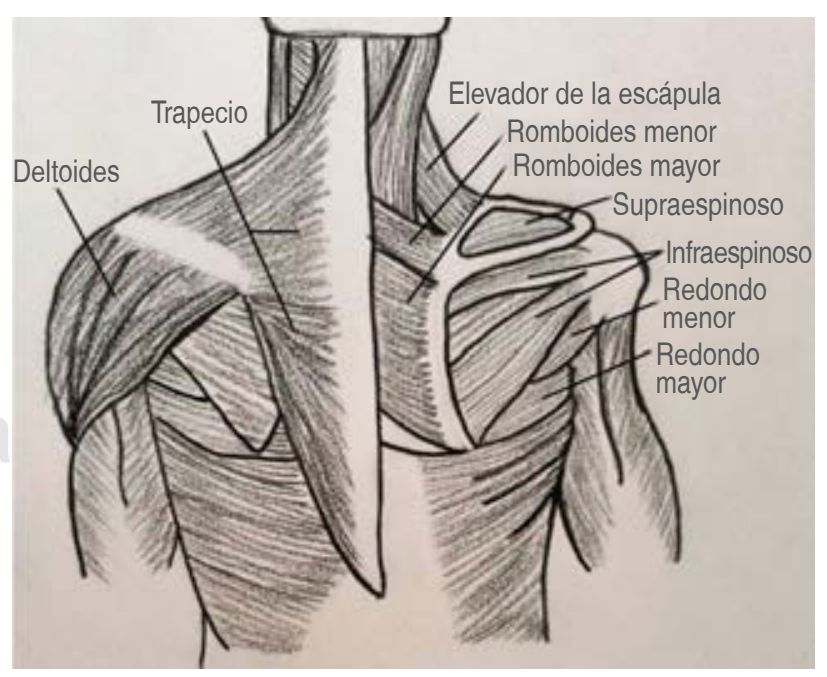

Figura 1: Principales músculos del hombro. Autor: Eliel Adonaí Magdaleno Navarro. 
traída e irreductible, por su tamaño se clasifica en primer grado $(<1 \mathrm{~cm})$, segundo grado, $(1-3 \mathrm{~cm})$, tercer grado, $(3-5 \mathrm{~cm})$ y cuarto grado $(>5 \mathrm{~cm}) \cdot{ }^{10}$

\section{Complicaciones y secuelas más frecuentes}

Sin tratamiento, la enfermedad del manguito rotador podría provocar rigidez o debilidad crónica y la posible degeneración progresiva de la articulación del hombro, además se puede engrosar y tensar el tejido conjuntivo que rodea la articulación (hombro rígido). Es necesario descansar el hombro para la recuperación y mantenerlo inmovilizado durante mucho tiempo. La probabilidad de una nueva rotura aumenta en función de la magnitud del daño previo: si el daño es muy acusado, la frecuencia de repetición del desgarre es de hasta $70 \% .^{11}$

\section{Estadísticas}

La patología del manguito rotador del hombro constituye un grupo de lesiones muy importantes en patología laboral o en el deporte, debido a su alta prevalencia e incidencia, tanto en el número de casos atendidos como en la frecuencia de aparición de secuelas, ya que constituyen la patología fundamental dentro de las afecciones de la articulación del hombro. ${ }^{12}$ Por ejemplo, en España 50\% de las personas mayores de 50 años presentan alguna lesión del manguito rotador y se estima que la prevalencia del dolor de hombro es de entre 16 y $26 \%$, con una incidencia anual de seis a 25 casos nuevos por cada 1,000 pacientes atendidos en un nivel de atención primaria. ${ }^{13}$

\section{Sintomatología de la lesión del manguito rotador}

El cuadro clínico de la patología del manguito rotador va asociada, fundamentalmente, a tres síntomas: dolor, debilidad y limitación de la movilidad. Los síntomas iniciales pueden ser leves, pero pueden evolucionar conforme la cronicidad de la lesión. Comúnmente se encontrará inflamación local a la presión en la parte frontal del hombro, el dolor se localiza en la región anterolateral y superior del hombro, y puede irradiarse a la región superior y externa del brazo, es típicamente nocturno y despierta al paciente. Durante el día es más tolerable, se desencadena con las actividades que requieren el uso del brazo por encima del nivel del hombro. La debilidad se manifiesta como fatiga o incapacidad para elevar el brazo por encima del nivel del hombro, lo cual limita las actividades de la vida diaria como el aseo, vestido, levantar objetos, entre otras. ${ }^{9,14}$

\section{Tratamientos}

El tratamiento a elegir depende de varios factores como el mecanismo, grado de lesión y el compromiso de otras estructuras. Hay dos tipos de tratamientos principales: el quirúrgico y el conservador, los cuales pueden ir acompañados o no de apoyo farmacológico. No obstante, hay evidencia limitada de que la cirugía no es más efectiva para tratar la rotura del manguito de los rotadores que el tratamiento conservador solo, ya que la fisioterapia es menos propensa a complicaciones y menos costosa que la cirugía, se recomienda un enfoque conservador como la modalidad de tratamiento inicial para las lesiones del manguito rotador.

Tratamiento quirúrgico: se indica cuando la lesión de tejidos blandos es masiva e implica un desgarro total del músculo o del tendón o está asociada a algún proceso en el cual esté afectada la circulación adyacente al hombro. ${ }^{9,14}$

Tratamiento conservador: es indicado en procesos con poca lesión de tejidos blandos como un pinzamiento o inestabilidad de hombro, o bien, en rupturas crónicas no traumáticas o que exista alguna contraindicación para comenzar el tratamiento quirúrgico. ${ }^{9,14}$ Se cuenta como tratamiento conservador al descanso, el hielo y la fisioterapia, con frecuencia es lo único que se necesita para recuperarse de una lesión del manguito rotador. ${ }^{9,14}$ 
Si los tratamientos conservadores no reducen el dolor se debe aplicar una inyección de esteroides en la articulación del hombro, especialmente si el dolor no permite el descanso, realizar actividades diarias o hacer ejercicio. A pesar de que esas inyecciones suelen servir, deben usarse con criterio, ya que pueden debilitar el tendón. Por otro lado, el tratamiento conservador consta de ejercicios de fisioterapia que pueden ayudar a restaurar la flexibilidad y la fuerza del hombro después de una lesión en el manguito de los rotadores. Algunas veces es posible eliminar el dolor y restablecer el funcionamiento sin cirugía, siendo la FNP uno de los tratamientos conservadores más prometedores. ${ }^{4}$

\section{Tratamiento del manguito rotador con} facilitación neuromuscular propioceptiva

La FNP tiene como objetivo enfocarse en aquello que el paciente puede hacer dentro de las limitaciones de la lesión. Es un concepto holístico e integra los aspectos sensoriales, motores y psicológicos de un programa de rehabilitación. Incorpora actividades reflejas de los niveles vertebrales y superiores, inhibiendo o facilitando según sea apropiado. ${ }^{15}$

La FNP establece que todos los seres humanos, incluyendo aquellas personas con discapacidades, tienen un potencial real sin explotar. Establece un abordaje positivo e integral, es decir, se enfoca a la persona en su totalidad y no en un solo segmento corporal o problema en específico. ${ }^{16}$ Se define a la FNP como un método que busca mejorar una respuesta muscular por medio de la vía neuromuscular, conformada de estímulos específicos por medio de la activación de los propioceptores. Su funcionalidad se encuentra basada en conocimientos de anatomía, neurofisiología y, sobre todo, de los movimientos normales del cuerpo humano. Desde 1947 este método se basa en los principios neurofisiológicos de Sherrington:

1. Postdescarga: sensación de aumento de fuerza después de la desaparición del estímulo facilitador.

2. Sumación temporal: aplicación de muchos estímulos débiles que se combinan en un pe- riodo de tiempo corto, inhibiendo o excitando una respuesta.

3. Sumación espacial: aplicación de estímulos con una duración corta, pero en diferentes áreas, que se combina para tener la misma respuesta que en la sumación temporal.

4. Irradiación: propagación del estímulo hacia zonas adyacentes como respuesta al desbordamiento y aumento de fuerza.

5. Inducción sucesiva: es la contracción muscular de agonistas seguida de la musculatura antagonista.

6. Inhibición recíproca: la contracción del músculo agonista debe ser sincronizada por músculos sinergistas y la inhibición del antagonista.

Cuando se utiliza la FNP se busca aprovechar la actividad refleja: un músculo que es estirado pasivamente al inicio del movimiento facilitará una contracción muscular más potente, realizando un patrón de movimiento en los tres planos anatómicos: plano sagital, plano coronal y plano transversal. El movimiento es realizado en forma de espiral y diagonal. ${ }^{17}$

Por lo tanto, y debido a la pérdida de rango de movimiento del hombro por la lesión de manguito rotador, la rehabilitación tiene como principal objetivo aumentar la amplitud de movimiento. Al ser la FNP un método que busca la participación activa del paciente, se logra más rápido dicho objetivo al involucrar mayor movimiento a la vez que el paciente aprende el movimiento correcto para poderlo realizar en su día a día y así evitar lesiones futuras, a diferencia de otros métodos de tratamiento en los que no se involucra la participación activa del paciente. A estas ventajas se les suma que no se requiere de aparatología, más que el terapeuta tenga los conocimientos esenciales de este método.

Por otro lado, al realizar la revisión bibliográfica, se logró encontrar solamente nueve artículos en los que se aplica este método: dos de Corea y USA, y uno de México, Brasil, Turquía, Polonia y España. En estos estudios se trabajó con un grupo de pacientes con alguna patología de hombro, así como en deportistas y personas sanas, a las que se les aplicó FNP y se compa- 
Tabla 1: Evidencia de la efectividad de la facilitación neuromuscular propioceptiva en la lesión del manguito rotador.

\begin{tabular}{|c|c|c|c|c|c|c|}
\hline & Pacientes & (n) Grupos de estudios & FNP & Parámetros evaluados & Eficiencia de FNP & Ref. País \\
\hline 1 & $\begin{array}{l}\text { Capsulitis } \\
\text { adhesiva }\end{array}$ & $\begin{array}{l}\text { (53) } \\
\text { 3 Grupos: } \\
\text { 1. FNP + FT } \\
\text { 2. Ejercicio + FT } \\
\text { 3. Sólo FT }\end{array}$ & $\begin{array}{l}\text { Sesión } \\
\text { única }\end{array}$ & $\begin{array}{l}\text { Dolor, disquinesia escapular, } \\
\text { rango de movimiento y } \\
\text { funcionalidad }\end{array}$ & $\begin{array}{l}\text { Mayor mejoría en el grupo } \\
\text { de FNP }\end{array}$ & $\begin{array}{c}15 \\
\text { Turquía }\end{array}$ \\
\hline 2 & $\begin{array}{l}\text { Lesión crónica } \\
>10 \text { años }\end{array}$ & (26) & 4 meses & $\begin{array}{l}\text { Movilidad, fuerza, } \\
\text { funcionalidad, dolor y } \\
\text { satisfacción }\end{array}$ & $100 \%$ en todas las variables & $\begin{array}{c}18 \\
\text { México }\end{array}$ \\
\hline 3 & $\begin{array}{l}\text { Ruptura de } \\
\text { supraespinoso }\end{array}$ & (20) & 3 meses & $\begin{array}{l}\text { Flujo sanguíneo, dolor, } \\
\text { funcionalidad }\end{array}$ & $\begin{array}{l}\text { Mejoría en la velocidad de } \\
\text { flujo sanguíneo }\end{array}$ & $\begin{array}{c}19 \\
\text { Corea }\end{array}$ \\
\hline 4 & $\begin{array}{l}\text { Personas } \\
\text { sanas, } \\
\text { ejercicio } 3 \\
\text { veces/semana }\end{array}$ & $\begin{array}{l}\quad(38) \\
2 \text { Grupos: } \\
\text { 1. FNP } \\
\text { 2. DUMBELL }\end{array}$ & $\begin{array}{l}\text { Sesión } \\
\text { única }\end{array}$ & $\begin{array}{l}\text { Ácido láctico, cortisol, } \\
\text { tiempo de contracciones } \\
\text { concéntricas y excéntricas } \\
\text { durante la elevación diagonal }\end{array}$ & $\begin{array}{l}\text { Promueve mayor } \\
\text { reclutamiento de los } \\
\text { músculos estabilizadores } \\
\text { dinámicos del hombro } \\
\text { durante los ejercicios }\end{array}$ & $\begin{array}{c}20 \\
\text { Brasil }\end{array}$ \\
\hline 5 & $\begin{array}{l}\text { Deporte de } \\
\text { lanzamiento } \\
\text { de mano }\end{array}$ & $\begin{array}{l}\text { (30) } \\
\text { 3 Grupos: } \\
\text { 1. CRC+ FNP } \\
\text { 2. HRC + FNP } \\
\text { 3. Control }\end{array}$ & $\begin{array}{l}2 \text { por } \\
\text { semanas } \\
\text { por } 6 \\
\text { semanas }\end{array}$ & $\begin{array}{l}\text { ROM para la rotación externa } \\
\text { del hombro }\end{array}$ & $\begin{array}{l}\text { Tanto CRC y HRC+PNF } \\
\text { permitieron aumentar el } \\
\text { ROM. }\end{array}$ & $\begin{array}{l}21 \\
\text { USA }\end{array}$ \\
\hline 6 & $\begin{array}{l}\text { Síndrome } \\
\text { de choque } \\
\text { subacromial }\end{array}$ & $\begin{array}{l}\quad(23) \\
\text { 2 Grupos: } \\
\text { 1. FNP } \\
\text { 2. Otro (láser, } \\
\text { terapia de campo } \\
\text { magnético y } \\
\text { crioterapia local) }\end{array}$ & $\begin{array}{l}\text { Sesión } \\
\text { única }\end{array}$ & $\begin{array}{l}\text { Medición de: rangos pasivos } \\
\text { y activos indoloros de } \\
\text { abducción, flexión y rotación } \\
\text { externa e interna de la } \\
\text { articulación del hombro }\end{array}$ & $\begin{array}{l}\text { Mejora el rango activo como } \\
\text { pasivo del movimiento el } \\
\text { hombro. Las terapias de } \\
\text { campo magnético con láser y } \\
\text { crioterapia local no mejoran } \\
\text { el rango de movimiento ni } \\
\text { disminuyen el dolor justo } \\
\text { después de una intervención. }\end{array}$ & $\begin{array}{c}22 \\
\text { Polonia }\end{array}$ \\
\hline 7 & $\begin{array}{l}\text { Tendinitis } \\
\text { calcificada }\end{array}$ & (1) & $\begin{array}{l}20 \text { sesiones } \\
40 \text { minutos } \\
\text { por } 2 \text { días }\end{array}$ & $\begin{array}{l}\text { Depósito de calcio } \\
\text { Escala analógica visual } \\
\text { Prueba de hombro simple } \\
\text { Escala de Constant-Murley } \\
\text { Rango de movimiento pasivo }\end{array}$ & $\begin{array}{l}\text { Disminuyeron los depósitos } \\
\text { de calcio. Redujo el dolor y } \\
\text { produjo efectos positivos en } \\
\text { la función del hombro. }\end{array}$ & $\begin{array}{c}23 \\
\text { Corea }\end{array}$ \\
\hline 8 & $\begin{array}{l}\text { Personas } \\
\text { sanas }\end{array}$ & $\begin{array}{l}\quad(25) \\
\text { 3 Grupos: } \\
\text { 1. Abducción } \\
\text { 2. FNP (D1F) } \\
\text { 3. FNP (D2F) + } \\
\text { levantamiento } \\
\text { de pesa }\end{array}$ & $\begin{array}{l}\text { Sesión } \\
\text { Única }\end{array}$ & $\begin{array}{l}\text { Patrones de activación } \\
\text { muscular }\end{array}$ & $\begin{array}{l}\text { Los seis músculos del } \\
\text { complejo del hombro } \\
\text { demostraron niveles de } \\
\text { activación muy altos }\end{array}$ & $\begin{array}{l}24 \\
\text { USA }\end{array}$ \\
\hline 9 & $\begin{array}{l}\text { Personas } \\
\text { mayores: } \\
\text { entrenamiento } \\
\text { de estiramiento }\end{array}$ & $\begin{array}{l}\quad(54) \\
\text { 3 Grupos: } \\
\text { 1. Pasivos } \\
\text { 2. FNP } \\
\text { 3. Controles }\end{array}$ & 13 semanas & Prueba ROM & $\begin{array}{l}\text { El aumento de ROM } \\
\text { fue similar en personas } \\
\text { físicamente activas similar } \\
\text { mediante FNP y las técnicas } \\
\text { pasivas }\end{array}$ & $\begin{array}{c}25 \\
\text { España }\end{array}$ \\
\hline
\end{tabular}


ró sus efectos con otros métodos conservadores. Todos los artículos describen que con la FNP los pacientes lograron tener una óptima recuperación y mejoría en parámetros como la disminución de movimiento, extensibilidad y funcionalidad del hombro (Tabla 1).

\section{CONCLUSIÓN}

La lesión del manguito rotador es una patología más frecuente en hombro. Hoy en día existen diferentes alternativas de tratamientos conservadores, entre las que se incluye FNP, la cual es un método conocido en el ámbito de la fisioterapia; no obstante, pese a sus buenos resultados, no existe suficiente evidencia que sustente su eficiencia en este tipo de lesiones, por lo que se recomienda promover más su aplicación, investigar y documentar adecuadamente para que un mayor número de personas puedan ser beneficiadas.

\section{BIBLIOGRAFÍA}

1. Ayala F, Sainz de Baranda P, Cejudo A. El entrenamiento de la flexibilidad: técnicas de estiramiento. Rev Andal Med Deporte. 2012; 5 (3): 105-112.

2. Bertinchamp U. Concepto FNP: facilitación neuromuscular propioceptiva (método Kabat-Knott-Voss). EMC-Kinesiterapia-Medicina Física. 2017; 38 (4): 1-13. http://dx.doi.org/10.1016/S1293-2965(17)87223-6.

3. Drake LR. Anatomía para estudiantes. 3era. ed. Madrid: Elsevier. 2015, p. 881.

4. Uglde-Ovares C, Zúñiga Mongue D, Barrentes Monge R. Actualización del síndrome de hombro doloroso: lesiones del manguito rotador. Med Leg Costa Rica. 2013; 30 (1): 63-71.

5. Suárez SN, Osorio PA. Biomecánica del hombro y bases fisiológicas de los ejercicios de Codman. Rev CES Med. 2013; 27 (2): 205-217.

6. Osma RJ, Carreño MF. Manguito de los rotadores: epidemiología, factores de riesgo, historia natural de la enfermedad y pronóstico. Revisión de conceptos actuales. Rev Colombiana de Ortopedia y Traumatología. 2016. 30 (1). 2-12. https://doi.org/10.1016/j.rccot.2016.09.001.

7. Bupa. Actividad y ejercicio lesión de manguito rotador. 2013 [Consultado 15/03/2020].

8. IMSS. Diagnóstico y tratamiento del síndrome de hombro doloroso en primer nivel de atención. Evidencias y Recomendaciones. Guía de Práctica Clínica GPC. México: Secretaría de Salud 2016. CENETEC.

9. IMSS. Diagnóstico y tratamiento del síndrome del manguito rotador. Guía de Práctica Clínica. MSS-617-13. México: Secretaría de Salud. 2013.

10. Nicolino TI, Paoletta R, Mecozzi G, Rossi LA, Bongiovanni S, Maigno G, Ranalletta M. Clasificación geométrica de rupturas del manguito rotador: excelente reproductibilidad y fiabilidad inter e intraobservador. Artroscopia. 2016; 23 (4): 136-140.

11. Gómez AM. El manguito de los rotadores. Ortho-tips. 2014; 10 (3): 144-153.

12. Sánchez-Sánchez F, Llinares Clausi BJ, Cruz Gisbert JM. Patología del manguito de los rotadores en el ambiente laboral. Master Universitario En Medicina Evaluadora. 2009, pp. 1-23.
13. González DR, Alamillo SJ, Gimenez-Moreno JL, Loscos-Gil P. Tratamiento rehabilitador tras la cirugía artroscópica del manguito rotador. Guías DNL. 2017, pp. 5-59.

14. Gómez-Acevedo JM. El manguito de los rotadores. Orthopics. 2014, 10 (3): 144-153.

15. Comuk BN, Ozlem YZ, Zeybek A, Gulsen M, Agah Tekindal M. Acute effect of scapular proprioceptive neuromuscular facilitation (PNF) techniques and classic exercises in adhesive capsulitis: a randomized controlled trial. J Phys Ther Sci. 2016; 28 (4): 1219-1227.

16. Garmendia P. Efectos de la FNP en la actitud postural en el paciente hemipléjico. Universidad Fasta. 2016.

17. Buck S. La facilitación neuromuscular propioceptiva en la Práctica. 3era. ed. California: Panamericana; 2012.

18. Jiménez AJ, Cortés SJ. Eficacia de la facilitación neuromuscular propioceptiva en el imbalance muscular de pacientes con síndrome de pellizcamiento. Rev Mex Ortop Traum. 1997; 11 (4): 274-278.

19. Kim JJ, Lee SY, Ha K. The effects of exercise using PNF in patients with a supraspinatus muscle tear. J Phys Ther Sci. 2015; 27 (8): 2443-2446. doi: 10.1589/ jpts.27.2443.

20. Comel JC, Nery RM, Garcia EL, da Silva Bueno C, de Oliveira Silveira E, Zarantonello MM, Stefani MA. A comparative study on the recruitment of shoulder stabilizing muscles and types of exercises. J Exerc Rehabil. 2018.26; 14 (2): 219-225. doi: 10.12965/ jer.1835198.599.

21. Decicco PV, Fisher MM. The effects of proprioceptive neuromuscular facilitation stretching on shoulder range of motion in overhand athletes. J Sports Med Phys Fitness. 2005; 45 (2): 183-187.

22. Olędzka M, Jaczewska-Bogacka J. Effectiveness of proprioceptive neuromuscular facilitation (PNF) in improving shoulder range of motion. A pilot study. Ortop Traumatol Rehabil. 2017; 19 (3): 285-292.

23. Oh DG, Yoo KT. The effects of therapeutic exercise using PNF on the size of calcium deposits, pain self-awareness, and shoulder joint function in a calcific tendinitis patient: a case study. J Phys Ther Sci. 2017; 29 (1): 163-167. doi: 10.1589/jpts.29.163. 
24. Youdas JW, Arend DB, Exstrom JM, Helmus TJ, Rozeboom JD, Hollman JH. Comparison of muscle activation levels during arm abduction in the plane of the scapula vs. proprioceptive neuromuscular facilitation upper extremity patterns. J Strength Cond Res. 2012; 26 (4): 1058-1065. doi: 10.1519/JSC.0b013e31822e597f.
25. González-Ravé JM, Sánchez-Gómez A, Santos-García DJ. Efficacy of two different stretch training programs (passive vs. proprioceptive neuromuscular facilitation) on shoulder and hip range of motion in older people. J Strength Cond Res. 2012; 26 (4): 1045-1051. doi: 10.1519/ JSC.0b013e31822dd4dd. 